
\title{
$\begin{array}{ll}\text { Research Square } & \begin{array}{l}\text { They should not be considered conclusive, used to inform clinical practice, } \\ \text { or referenced by the media as validated information. }\end{array}\end{array}$
}

\section{miR-138 from Adipose-Derived Stem-Cell Exosomes Accelerates Wound Healing in Diabetic Rats Through Targeting SIRT1/PTEN Signaling to Promote Angiogenesis and Fibrosis}

\author{
Xue Li \\ Shanghai Tenth People's Hospital \\ Nan Hu \\ Nanjing University Medical School Affiliated Nanjing Drum Tower Hospital \\ Miaomiao Zhang \\ Tongren Hospital Shanghai Jiaotong University School of Medicine \\ Jianhong Han \\ Shanghai Tenth People's Hospital \\ Yi Sun \\ Tongren Hospital Shanghai Jiaotong University School of Medicine \\ Hualan Yang \\ Shanghai Pudong Hospital \\ Maoquan Li (D maoquan201609@163.com) \\ Shanghai Tenth People's Hospital
}

Research Article

Keywords: miR-138, ADSCs, Exosome, SIRT1, cutaneous wound healing.

Posted Date: June 1st, 2021

DOI: https://doi.org/10.21203/rs.3.rs-521781/v1

License: (9) (7) This work is licensed under a Creative Commons Attribution 4.0 International License. Read Full License 


\section{Abstract}

Background: Exosome (Exo) secretion by adipose-derived mesenchymal stem cells (ADSCs) promotes cutaneous wound healing by transfer of bioactive molecules. miR-138 is a micro (mi)RNA that stimulates endothelial progenitor cells and promotes proliferation and locomotion of human scar fibroblasts (HSFs). However, the underlying molecular mechanism is unclear.

Methods: miR-138 activity in Exo-mediated healing was investigated by subcutaneous injection of exosomes isolated with ultracentrifugation from control ADSCs or ADSCs expressing miR-138 into full-thickness skin wounds in a rat diabetes model. Wound healing was evaluated by wound closure rate, histological evaluation, and immunofluorescence staining. Cultures of HSFs and human mammary epithelial cells (HMECs) were treated with Exos from wildtype and miR-138-modified ADSCs under high glucose conditions (HG). Cell proliferation and apoptosis were measured with cell counting kit (CCK)-8 assay and flow cytometry. Cell migration was assayed in Transwell chambers, and the effect of miR-138 on Exo-mediated angiogenesis and protein expression were evaluated. The miR-138 target was identified with a luciferase reporter assay.

Results: ADSC-Exos were incorporated by endothelial cells and HSFs, and miR-138 enhanced cell proliferation and migration and suppressed apoptosis under HG conditions. Exos promoted endothelial tubule formation by HSFs, and western blotting showed that miR-138 mediated the therapeutic effects by reversing SIRT1/PTEN-mediated PI3K/Akt and ERK1/2 signaling inhibition. The luciferase reporter assay confirmed that miR-138 interacted with the 3'-UTR of SIRT1 to suppress SIRT1 mRNA expression. Injection of miR-138-enriched Exos into skin wounds accelerated re-epithelialization, reduced scar width, and enhanced angiogenesis.

Conclusions: In conclusion, miR-138-enriched Exos promoted wound healing in a rat diabetes model by targeting SIRT1, and their effects in promoting soft tissue wound healing warrant further study.

\section{Introduction}

Dermatological diseases occur frequently in patients with diabetes and can manifest as fungal and bacterial and infections, granuloma annulare, diabetic dermopathy, or necrobiosis lipoidica diabeticorum [1,2]. Clinical applications of regenerative treatments with growth factors and other cell therapies have been successful in treating diabetes-associated skin injuries, including diabetic foot ulcers [3].

Mesenchymal stem cells (MSCs) in normal skin take part in wound repair, and adipose-derived mesenchymal stem cells (ADSCs) have been verified to improve wound healing in diabetic rats [4-6]. Angiogenesis has crucial functions in wound healing, and chronic wounds in diabetes are characterized by decreased angiogenesis [7]. We have previously reported that ADSCs exosomes enhance wound healing and prevent injury to cells transplanted into a high glucose environment [8].

Exosomes are extracellular vesicles of 30-150 nm that are secreted into the extracellular milieu by a majority of cell types. They have been investigated in fluids including blood, semen, urine, and the tissue extracellular matrix. They carry cargo, which participate in mediating interactions among organs and cells $[9,10]$. Exosomes have physiological activities relevant in both health and disease [11, 12]. Exosomes have been found to be paracrine mediators that induce healing by transferring bioactive molecules, including micro (mi)RNAs, proteins and other compounds [13, 14]. The ADSCs protective effects in wound healing have been suggested to be modulated by exosomes. ADSC exosomes can deliver miRNAs, which then ameliorate the high glucose environment in diabetes mellitus.

miRNAs are 19-25 nucleotide noncoding RNAs that suppress post-transcriptional gene expression through imperfect complementary sequence pairing at their target genes' 3 ' untranslated regions (3'UTRs), thereby causing translational repression or mRNA degradation [15, 16]. miRNAs have been demonstrated to participate in regulating $33.3 \%$ of all human genes, and thus, are involved in many biological functions $[17,18]$. Several studies have suggested that miRNAs regulate endothelial progenitor cell functions and may be novel therapeutic options for treating dermatological diseases.

miR-138 regulates cell proliferation, differentiation, and apoptosis [19]. It has been shown to enhance endothelial progenitor cell activity and human scar fibroblast (HSF) proliferation and locomotion [20,21], possibly by suppressing Sirtuin type 1 (SIRT1) expression [22, 23]. miR-138 is known to significantly decrease in type 2 diabetes mellitus (40), but the underlying mechanism is unclear.

SIRT1 is a silent information regulator 2 (Sir2) mammalian homolog that modulates wound healing. In diabetes [24, 25], SIRT1 deficiency inhibits PTEN expression, which enhances the PI3K/Akt and ERK signaling activation [26]. ERK and PI3K/Akt signaling have been verified to promote cutaneous wound healing [27-29]. We established that the exosomes secreted by miR-138-modified ADSCs have therapeutic effects in angiogenesis and fibroblast function, in vitro, under high-glucose conditions and on cutaneous wound healing in a rat diabetes model. miR-138's activity in promoting exosome-mediated wound healing was investigated.

\section{Materials \& Methods}

\section{Animals and ethics statement}

The Animal Care and Utilization Committee of the Shanghai Tenth People's Hospital approved all animal procedures. We obtained male Sprague-Dawley female rats at eight weeks of age from the SLAC Laboratory Animal Co. Ltd, Shanghai, China, and individually housed them in independent ventilated cages under $24^{\circ} \mathrm{C}$ to $26^{\circ} \mathrm{C}$ under constant humidity with a 12-hour light/dark cycle. All procedures were approved by the Ethics Committee of the the Shanghai Tenth People's Hospital, Shanghai, and were conducted by following the guidelines. We conducted surgical processes under anesthesia and made every effort to

Loading [MathJax]/jax/output/CommonHTML/fonts/TeX/fontdata.js 
minimize suffering. We anesthetized rats by intraperitoneal injection of $30 \mathrm{mg} / \mathrm{kg}$ sodium pentobarbital before sacrifice and the rats were sacrifice by spinal dislocation.

\section{ADSC isolation, culture, and identification}

Briefly, we harvested adipose tissue from normal rats or healthy humans. The Ethics Committee of the Shanghai Tenth People's Hospital, Shanghai approved all procedures (Human20170621 for humans, Animal20170513 for animals). We conducted procedures by following the guidelines. We conducted surgical processes under anesthesia and made every effort to minimize suffering. We washed tissues with phosphate-buffered saline (PBS) and minced them before digestion with $0.2 \%$ collagenase I (Sigma-Aldrich, St. Louis, USA) for 1 hour at $37^{\circ} \mathrm{C}$ with intermittent shaking. We washed the digested tissue with Dulbecco's modified Eagle's medium (DMEM) (Sigma-Aldrich, St. Louis, USA) containing 15\% FBS (Gibco BRL, Frederick, USA) and centrifuged the samples at 1000 rpm for 10 minutes to remove mature adipocytes. We resuspended the pellets in DMEM with $15 \% \mathrm{FBS}$, with penicillin (100 $\mathrm{U} / \mathrm{ml})$ and streptomycin (100 $\mu \mathrm{g} / \mathrm{ml})$, and cultured cells at $37{ }^{\circ} \mathrm{C}$ and $5 \% \mathrm{CO}_{2}$. We detached $\mathrm{ADSC}$ reaching $80 \%-90 \%$ confluence with $0.02 \%$ ethylenediaminetetraacetic acid/0.25\% trypsin (Sigma-Aldrich, St. Louis, USA) for 5 minutes at room temperature and replated them. We utilized fluorescein isothiocyanate (FITC)- or phycoerythrin (PE)conjugated antibodies against CD29, CD44, CD90, CD105, and von Willebrand factor (vWF) for phenotypic analysis. We used IgG-matched isotype as the internal control for each antibody. We grew normoxic ADSC cultures in $95 \%$ air $\left(20 \% \mathrm{O}_{2}\right)$ and $5 \% \mathrm{CO}_{2}$.

\section{Multilineage differentiation of ADSCs}

To validate ADSC multilineage differentiation, we cultured third-passage mouse ADSCs in adipogenic differentiation medium (Sigma-Aldrich, St. Louis, USA) and stained them with oil red $\mathrm{O}$ after 2 weeks, or cultured them in osteogenic differentiation medium (Sigma-Aldrich, St. Louis, USA) and stained them with alizarin red after 3 weeks.

\section{ADSC-derived exosome isolation and identification}

After reaching $80 \%-90 \%$ confluence, ADSCs were rinsed with PBS and cultured in FBS-free endothelial cell growth medium-2MV, supplemented with $1 \times$ serum replacement solution for another 2 days. We removed conditioned culture medium and centrifuged the cells at $300 \mathrm{~g}$ for $10 \mathrm{minutes}$ and at another $2000 \mathrm{~g}$ for 10 minutes to remove cellular debris and apoptotic cells. After centrifugation at $10,000 \mathrm{~g}$ for 0.5 hour, we filtered the supernatant ( $0.22 \mu \mathrm{m}$, Millipore, Billerica, USA), transferred $15 \mathrm{~mL}$ of supernatant to an Amicon Ultra-15 Centrifugal Filter Unit (100 kDa, Millipore) and centrifuged it at $4000 \mathrm{~g}$ to concentrate the sample to $\sim 1 \mathrm{ml}$. We washed the ultrafiltration unit twice with PBS and filtered the samples again at $4000 \mathrm{~g}$ to achieve a $1 \mathrm{ml}$ volume. We added a $20 \%$ volume of Exoquick exosome precipitation solution (System Biosciences, USA) to the ultrafiltered liquid and mixed the sample through inversion. After incubation for half a day, we centrifuged the mixture at $1500 \mathrm{~g}$ for 0.5 hour and aspirated the supernatant. We resuspended the exosome pellets in $500 \mu \mathrm{L}$ PBS. We performed all procedures at $4^{\circ} \mathrm{C}$. We determined the exosome protein content with a Pierce bicinchoninic acid Protein Assay Kit (Thermo Fisher Scientific, USA). We stored exosomes at $-80^{\circ} \mathrm{C}$ until use. We utilized western blotting and transmission electron microscopy to analyze the exosomes. We determined size by dynamic light scattering with a Nanosizer. The size distribution was plotted with the particle radius (nm) on the $X$-axis and the percentage on the $Y$-axis.

\section{Bioinformatics analysis}

The miRNA target of SIRT1 were predictions with the targetscan (http://www.targetscan.org/).

\section{Cell culture}

We cultured HSFs and HEK293T (FuHeng Biology, Shanghai, China) in high glucose DMEM (Gibco BRL, Grand Island, USA) with 10\% FBS. We cultured human mammary endothelial cells (HMECs, Cell Bank of the Chinese Academy of Sciences, Shanghai, China) in MCDB131 medium (Gibco BRL) containing 10\% FBS, $2 \mathrm{mM}$ L-glutamine (Sigma-Aldrich), epidermal growth factor (Sigma-Aldrich, $10 \mathrm{ng} / \mathrm{ml}$ ) and hydrocortisone (1 $\mu \mathrm{g} / \mathrm{ml}$, Sigma-Aldrich). We maintained cells under $37^{\circ} \mathrm{C}$ and $5 \% \mathrm{CO}_{2}$ in a humidified environment. HMECs and HSFs were stimulated with $5.5 \mathrm{mM}$ or $30 \mathrm{mM}$ glucose for $1 \mathrm{day}$, and $100 \mu \mathrm{g} / \mathrm{ml}$ of exosomes was added to the cultures to assess protection against high glucose injury.

\section{Exosome labeling and uptake}

We used a PKH67 fluorescent linker kit (Sigma-Aldrich, St. Louis, USA) to label exosomes. We added PKH67 dye (400 $\mu \mathrm{L})$ to exosome suspensions and incubated the samples for 5 minutes at room temperature. We added an identical volume of exosome-depleted bovine serum albumin to stop the reaction, and washed exosomes twice with PBS to remove any unbound dye. We incubated HSFs or HMECs with exosomes labeled for 3 hours, fixed and stained samples with diamidinophenylindole (DAPI), and observed and photographed them under a confocal microscope.

\section{RNA interference or overexpression}

We purchased miR-138 inhibitors from RiboBio (Guangzhou, China), and conducted transfection according to standard procedures. In brief, we transferred cells to culture plates with six wells and transfected them through incubation in complete medium containing ADSC-exosomes (200 $\mu \mathrm{g} / \mathrm{well}$, at $100 \mu \mathrm{g} / \mathrm{ml})$ and Lipofectamine 2000 (Thermo Scientific), or an equivalent volume of PBS for 1 day. For miR-138 overexpression, ADSCs were transfected with an miR-138 mimic (5'-AGCGUGUGUUGUGAAUCAGGCCG-3') synthesized by GenePharma (Shanghai, China) by Lipofectamine 2000, as described previously.

\section{Quantitative real-time polymerase chain reaction (qPCR)}


We extracted RNAs from cells with a TRIzol reagent kit (Invitrogen, Carlsbad, USA), by following the standard procedures. We amplified cDNAs and performed reverse transcription with a TaqMan miRNA reverse transcription kit. The primers used to assay SIRT1 (Gene ID: 309757) expression were reverse, 5'-

CACTTTCATCTTCCAAGGGTTC-3' and forward, 5'-TATTCCACGGTGCTGAGGTA-3'. The PTEN (Gene ID: 50557) primers were reverse, 5'-

AAGAGTCAAACAATGGCAAGC-3' and forward, 5'-TAGTTGGAGTCACCAGGATG-3'. The miR-138 (accession number: MIMAT0000844) primers were reverse, 5'CTCAACTGGTGTCGTGGAGTCGGCAATTCAGTTGAGCGGCCTGATTC-3' and forward, 5'-ACACTCCAGCTGGGAGCTGGTGTTGTGTTG-3'. qPCR was conducted with a TaqMan human miRNA assay kit. We used $2^{-\triangle A C T}$ method to capture relative fold differences in expression. U6 and $G A P D H$ were used as internal references.

\section{Flow cytometry}

We used flow cytometry to assay HMECs apoptosis after FITC-conjugated annexin $\mathrm{V}$ and propidium iodide (PI) staining. We washed cells twice before adjusting them to $1 \times 10^{6}$ cells per $\mathrm{ml}$ in cold D-Hanks buffer. We added annexin V-FITC (10 $\left.\mu \mathrm{l}\right)$ and PI (10 $\left.\mu \mathrm{l}\right)$ to $100 \mu \mathrm{L}$ cell suspension and incubated them for 15 minutes at room temperature in the dark. Before analysis, we added $400 \mu$ l binding buffer to each sample without washing. We performed each assay in at least triplicate.

\section{Tubule formation assay}

In vitro neovascularization was assayed in matrices of human fibrin. After treatment, we seeded serum-starved HMECs in endothelial basal medium onto plates coated with Matrigel ( $10^{5}$ cells per well into six well plates) (BD Biosciences, Franklin Lakes, USA) and incubated them at $37^{\circ} \mathrm{C}$ for 12 hours. We observed tubular structures that formed in the Matrigel and photographed under phase-contrast microscopy, and the newly formed tube lengths in ten randomly selected fields per well were measured.

\section{Transwell assay}

We treated cells with exosomes, miR-138-exosomes, or miR-138 inhibitor under HG conditions. After $24 \mathrm{~h}$, we starved the cells in serum-free medium for another 12 hours, performed trypsin digestion, and seeded $1 \times 10^{5}$ cells in the top chambers of 24 well Transwell culture inserts (Promega, Fitchburg, WI, USA). Medium with $20 \%$ serum was used as a chemoattractant. We fixed the cells for 10 min with formalin of $4 \%$ after $24 \mathrm{~h}$ culture.

\section{Cell counting kit (CCK)-8 assay}

We assayed HSF and HMEC proliferation with a CCK-8 kit (BD Biosciences, Franklin Lakes, USA) by using standard procedures. We cultured transfected cells in 96-well plates with exosomes under HG conditions for $24 \mathrm{~h}$ before adding $90 \mu \mathrm{L}$ fresh culture medium and $10 \mu \mathrm{l}$ CCK-8 reagent. We detected the absorbance at $450 \mathrm{~nm}$ with a microplate reader after incubation at $37^{\circ} \mathrm{C}$ for 2 hours.

\section{Luciferase reporter assay}

The 3'-UTR target sequence for miR-138 miRNA in the SIRT1 gene was predicted with the argetScan online tool. Wild-type and 3'-UTR mutant SIRT1 were performed and cloned into the pMIR firefly luciferase-expressing vector. We cotransfected HEK293T cells at $70 \%$ confluence with 500 ng of pMIR-SIRT1wt/pMIR-SIRT1-Mut and $50 \mathrm{nM}$ of miR-138 mimics with a Lipofectamine 2000 transfection kit (Thermo Scientific) for the luciferase assays. We assayed luciferase activity with the Dual-Luciferase Reporter System (Promega). We independently performed five assays.

\section{Rat diabetes skin wound model}

We induced diabetes in rats through a single intraperitoneal injection of $100 \mathrm{mg} / \mathrm{kg}$ in $0.01 \mathrm{M} \mathrm{pH} 4.3$ sodium citrate. We measured the blood glucose daily and controlled it to between 16.7 and $33.3 \mathrm{mmol} / \mathrm{I}$ through administration of insulin at 6-18 U/day (Wan-Bang Biochemical Medicine Co. Ltd, Xuzhou, China). After 1 month, we established the subcutaneous wound model after isoflurane inhalation anesthesia (2\%). We made a single round full-thickness skin wound on the dorsal hind foot with a disposable $5 \mathrm{~mm}$ skin biopsy punch and Westcott scissors. Eighteen rats were allocated randomly to subcutaneously injection of $200 \mu \mathrm{g}$ ADSC-exosomes in PBS of $100 \mu \mathrm{l}$ or the same volume of PBS at four sites around the wound ( $25 \mu \mathrm{l}$ per site). The rats were killed after 15 days, and skin specimens were harvested for histopathological evaluation. Each group have 6 rats. We anesthetized rats by intraperitoneal injection of $30 \mathrm{mg} / \mathrm{kg}$ sodium pentobarbital before sacrifice and the rats were sacrifice by spinal dislocation.

\section{Measurement of wound contraction}

On days 0,7 , and 15 before wound harvest, the sizes of the treated and control wounds were measured with a ruler and photographed (DMC-LX5GK, Panasonic, Japan). We calculated the ulcer area with Image-Pro Plus 4.5 (http://www.mediacy.com/imageproplus).

\section{Immunohistochemistry and immunofluorescenceassays}

We fixed skin tissue samples in 10\% formalin solution, embedded them in paraffin, and sectioned them at $5 \mu$ m. We stained tissue sections with Masson's trichrome for histological evaluation. We performed immunofluorescence staining of CD31 to measure histopathological alterations in angiogenesis and used terminal deoxynucleotidyl transferase dUTP nick end labeling (TUNEL) to detect apoptotic cells. We examined sections with a fluorescence microscope (Nikon, Tokyo, Japan) or an Axiophot light microscope (Zeiss, Oberkochen, Germany) and photographed them with a digital camera.

\section{Western blot assay}


We lysed tissues or cells, and centrifuged lysates at $12,000 \mathrm{rpm}$ under $4^{\circ} \mathrm{C}$ after adding a protease inhibitor. We determined protein concentrations via a Pierce bicinchoninic acid assay kit (Thermo Fisher). We separated proteins by $10 \%$ SDS-PAGE and transferred them to PVDF membranes. To assay protein expression, we used primary antibodies against CD63 (1:600), CD9 (1:600), CD31 (1:1000), CD81 (1:600), SIRT1 (1:500), CD34 (1:1000), PTEN (1:500), p-Akt (1:500), Bax (1:200), caspase-3 (1:600), p-ERK1/2 (1:500), Akt (1:500), ERK1/2 (1:500, all Santa Cruz Biotechnology, Dallas, USA), and GAPDH (1:1000, SigmaAldrich). We also used horseradish peroxidase-conjugated secondary antibody (1:1000, Abcam, USA). We used an ECL chemiluminescent kit (Millipore, Burlington, MA, USA) to detect bands.

\section{Statistical analysis}

We denoted continuous variables as means \pm SD (standard deviation). We performed one-way analysis of variance for comparisons in GraphPad Prism (GraphPad, La Jolla, USA). A p-value $\leq 0.05$ was considered to indicate a significant difference.

\section{Results}

\section{Characterization of ADSC-exosomes}

We previously suggested that exosomes participate in mesenchymal stem cell-mediated wound healing in a diabetes foot ulcer model [7]. Exosome transfer of erythroid 2-related factor 2 (Nrf2) nuclear factor accelerated the cutaneous wound healing by promoting vascularization. miR-138 has been reported to enhance endothelial progenitor cell function in a mouse venous thrombosis model [20]. The involvement of miR-138 in exosome-mediated wound healing was confirmed in ADSCs isolated from human cell lines and rat adipose tissue. The isolated ADSCs had a typical cobblestone-like morphology (Fig. 1A). Immunofluorescence staining was positive for the expression of surface mesenchymal cell markers including CD29, CD90, CD44, and CD105, but negative for the endothelial markers CD34 and vWF (Fig. 1B-H). The oil red $\mathrm{O}$ and alizarin red staining data verified adipocyte differentiation and osteoblast differentiation (Fig. 1l, J).

Dynamic light scattering showed that the particle diameters ranged from 50 to $120 \mathrm{~nm}$ (Fig. 1K), in agreement with the distributions of exosome size reported in [30]. The ADSC-exosomes had cup- or sphere-shaped morphologies in transmission electron micrographs (Fig. 1L), similar to previously described exosomes [30]. The expression of exosome marker proteins such as CD81, CD63, CD31, and CD9 in ADSC-exosomes was confirmed with western blotting (Fig. $1 \mathrm{M})$. The data demonstrated that both ADSC-exosomes and cellular components expressed CD63, CD81 CD31, and CD9, and that the nanoparticles were exosomes.

\section{ADSC-exosomes transfer miR-138 into fibroblasts and endothelial cells}

ADSCs overexpressing miR-138 were constructed to investigate the exosome-mediated promotion of wound healing. qPCR analysis confirmed miR-138 overexpression in the transformed ADSCs, as compared with wild-type ADSCs (Fig. 2A). To determine whether ADSC-exosomes were internalized by endothelial cells and fibroblasts, a prerequisite for subsequent exosomal miRNA transfer, we incubated ADSC-exosomes labeled with the green fluorescent dye PKH67 with HSFs and HMECs, and washed them to remove unbound exosomes, and fixed them. We stained DNA with DAPI. Fluorescence microscopy demonstrated that PKH67-labeled exosomes entered into HSFs and HMECs (Fig. 2B), and qPCR confirmed the presence of miR-138 in the recipient cells after culture with ADSC-exosomes. miR-138 expression increased in both HSFs and HMECs after treatment with exosomes, and in ADSCs transfected with miR-138, as compared with wild-type ADSCs (Fig. 2C, D). qPCR confirmed that binding of miR-138 to the SIRT13'-UTR downregulated the mRNA and protein expression of SIRTI and its downstream gene PTEN in both HSFs and HMECs after exosome treatment, compared with controls (Fig. 2E-H).

\section{miR-138 is involved in ADSC-exosome-mediated angiogenesis, migration, and inhibition of apoptosis of HMECs in high-glucose cultures}

HMECs were cultured in medium with $30 \mathrm{mM}$ glucose and PBS, exosomes, miR-138-exosomes, or miR-138-exosomes plus miR-138 inhibitor for 24 h. The Transwell assays indicated that high glucose inhibited HMEC migration, but exosome treatment reversed the effect. miR-138 overexpression in exosomes increased the recovery of HMEC migration, but miR-138 inhibitor treatment significantly inhibited the protective effect of exosomes (Fig. 3A, B). Flow cytometry analysis revealed that miR-138-exosome treatment significantly suppressed the apoptosis of HMECs under high glucose and that miR-138 inhibitor treatment reversed the protective effect of exosomes (Fig. 3C, D). Thus, miR-138 appears to have a protective effect against high glucose induced apoptosis in HMECs. The formation of tubules by HMECs on Matrigel-coated culture wells was used as an in vitro model of angiogenesis, as assessed by the number of branches that formed. miR-138-exosomes reversed the inhibition of angiogenesis in response to high glucose, an effect that was blocked by miR-138 inhibitor treatment (Fig. 3E, F). The co-culture assay between ADSCs and HMECs found that miR-138-modified ADSCs promotion angiogenesis of HMECs under HG condition, but after added the ADSCs exosome inhibitor, the promotion effect was reversed (Fig. S1). Suggestion that exosomes play a mediating role.

CCK-8 assays confirmed that high glucose conditions decreased cell proliferation and that exosomes, especially miR-138-exosomes, promoted HMEC proliferation. miR-138 inhibitor cotreatment decreased the effects of exosomes on HMEC proliferation (Fig. 3G).

qPCR indicated that miR-138-exosome treatment suppressed the SIRT1 and PTEN mRNA expression due to high glucose and that cotreatment with the miR138 inhibitor reversed the miR-138-exosome effect (Fig. 3H, I). The co-culture assay between ADSCs and HMECs found that miR-138-modified ADSCs promotion angiogenesis of HMECs under HG condition, but after added the ADSCs exosome inhibit the promotion effect was reversed (Fig. $3 \mathrm{~J}$ ), suggestion that the angiogenesis promotion effect of miR-138 was ADSC-exosome mediated. The in vitro functional assays in HMEC cultures indicated that miR-138 is involved in ADSC-exosome-mediated activation and the promotion of angiogenesis, cell migration, and activation, as well as the inhibition of apoptosis, possibly by targeting SIRT1/PTEN signaling. 
The effects of ADSC-exosomes on HSFs were evaluated in cultures with $30 \mathrm{mM}$ glucose, which were treated with PBS, exosomes, miR-138-exosomes, or miR138-exosomes plus miR-138 inhibitor for $24 \mathrm{~h}$. High glucose inhibited HFS migration in Transwell chambers, an effect reversed by exosome treatment. miR138 overexpression increased the recovery of HSF migration, although the effect was largely reversed by miR-138 inhibitor treatment (Fig. 4A, B). Flow cytometry analysis indicated that miR-138-exosome treatment protected against high glucose-induced HSF apoptosis and that miR-138 inhibitor treatment reversed the protective effect (Fig. 4C, D). The CCK-8 assays indicated that high glucose conditions inhibited cell proliferation and that treatment with exosomes and 138-exosomes enhanced the proliferative activity of HSFs cultured in high glucose medium. Cotreatment with miR-138 inhibitor interfered with the effects of exosome treatment (Fig. 4E). The qPCR results showed that miR-138-exosome treatment suppressed the expression of SIRT1 and PTEN mRNA occurred due to high glucose, and that cotreatment with miR-138 inhibitor reversed the effect (Fig. 4F, G). In vitro functional assays of HSF cultures indicated that miR-138 was involved in ADSC-exosome-mediated activation, the promotion of cell migration, and apoptosis inhibition, possibly through targeting SIRT1/PTEN signaling. The RT-qPCR detection also found that miR-138 expression in ADSCs were decreased after transfected with inhibitor, but increased after transfected with mimic. The result also show that miR-138 overexpression inhibit SIRT1 and PTEN expression. Inhibit miR-138 expression promotion SIRT1 and PTEN expression (Fig. 4H-M).

\section{Akt and ERK1/2 phosphorylation is enhanced by miR-138 transfer from ADSC-exosomes}

ERK1/2 and PI3K/Akt pathway activation occurs during cutaneous wound healing [31, 32]. miR-138 promotes ERK1/2 and PI3K/Akt activation [33, 34]. In this research, ADSC-exosomes were used to deliver miR-138 to activate those signaling pathways in HMECs (Fig. 5A-C) and HSFs (Fig. 5D-F) cultured in high glucose media. Treatment with miR-138 inhibitor suppressed the effects of miR-138, thus, suggesting that activation of ERK1/2 and PI3K/Akt might be mediated by SIRT1 inhibition. The western blot results indicated that miR-138-exosomes suppressed the pro-apoptosis proteins, caspase-3 and Bax in HMECs (Fig. 5G-I) and HSFs (Fig. 5J-L) in high glucose cultures. The miR-138 inhibitor suppressed the exosome's effects on protein expression involved in apoptosis. Bioinformatics analysis (http://www.targetscan.org/) found that SIRT1 can interacted with miR-138, miR-384, mir-1257, miR-30, miR-138, miR-141, miR-200, miR-217 and so on. Then we constructed the Luciferase reporter analysis vector by insert the sequence of SIRT1 3'UTR. After transfected with Luciferase reporter analysis vector combine with different miRNA mimic. The result show that only miR-138 can suppressed luciferase activity. Suggestion that miR-138 was the target of SIRT1. The dual-luciferase reporter results further confirmed that miR-138 interacted with the SIRT1 3'-UTR (Fig. 6A) and suppressed SIRT1 mRNA expression (Fig. 6B). SIRT1 mRNA and protein expression was significantly downregulated in HMECs (Fig. 6C) and HSFs (Fig. 6D) transfected with an miR-138 mimic, but increased after transfected with an miR-138 inhibitor, as compared with control or negative control cells. The data indicated that miR-138's protective effect against high-glucose-induced cell damage was associated with SIRT1 inhibition.

\section{Exosomes from miR-138-modified ADSCs accelerate cutaneous wound healing in diabetic rats}

We investigated ADSC-exosomes' effects on wound healing in full-thickness cutaneous wounds in the feet of rats with streptozotocin-induced diabetes in response to subcutaneous injection of equivalent volumes of exosomes, miR-138-exosomes, or PBS exosome diluent. Wound closure was significantly accelerated by miR-138 exosomes, compared with the control PBS treatment. The wounds treated with miR-138-exosomes were nearly closed on day 14 , but large scar areas were visible in control wounds (Fig. 7A, B). Masson-stained tissue from wounds treated with miR-138-exosomes included more collagen fibers than tissue from controls after 14 days (Fig. 7C, D). CD31 staining indicated that microvascular development was significantly more extensive after treatment with exosomes and miR-138-exosomes than after the control treatment (Fig. 7E, F). TUNEL staining indicated that miR-138-exosome treatment significantly suppressed apoptosis of skin tissue, as compared with control treatment (Fig.7G, H). Western blotting confirmed that miR-138-exosome treatment strongly suppressed apoptosis protein expression, including that of caspase-3 and Bax (Fig. 7I-K), and SIRT1 and PTEN (Fig. 7L-N), as compared with control treatment.

\section{Discussion}

This study provides the first demonstration of miR-138 activity in ADSC-exosome-mediated wound healing. The local transplantation of exosomes from ADSCs overexpressing miR-138 into skin wounds shortened the time of wound closure and increased the rates of collagen deposition, re-epithelialization, and new blood vessel formation. Scar formation was decreased. ADSC-exosomes were internalized, and miR-138 was transferred to both fibroblasts and endothelial cells. The upregulation of miR-138 expression activated and enhanced the migration of both fibroblasts and endothelial cells and suppressed apoptosis after exposure to high glucose culture conditions. Previous studies have found that up-regulation of miR-138 inhibits hypoxia-induced cardiomyocyte apoptosis via down-regulating lipocalin-2 expression [35]. miR-138 protects cardiomyocytes from hypoxia-induced apoptosis via MLK3/JNK/cjun pathway [36]. In this study, we found that transfer of miR-138 to endothelial cells by ADSC-exosomes enhanced cultured endothelial cell angiogenesis. Bioinformatics and a luciferase reporter assay confirmed that miR-138 interacted with SIRT1 and decreased the expression of SIRT1 mRNA and protein, which were associated with decreased PTEN expression. The observed inhibitory effect of SIRT1/PTEN on ERK1/2 and PI3K/Akt signaling is consistent with findings from previous reports [26].

Exosomes in human umbilical cord blood-derived epithelial progenitor cells (hucMSC-exo) have been found to promote wound healing in rats with streptozotocin-induced diabetes through enhancing angiogenesis via Erk1/2 signaling [27]. Akt pathway activation by hucMSC-Exo is also associated with a heat stress-induced decrease in apoptosis in a rat skin burn model [37]. PTEN is a PI3K/Akt pathway negative regulator that acts by dephosphorylating PIP3 (phosphatidylinositol 5, 4, 3 phosphate) to PIP2 (phosphatidylinositol 5, 4 phosphate) [38, 39]. It suppresses the activation of ERK1/2 [40, 41], and PTEN overexpression promotes apoptosis under stress conditions [42]. SIRT1 regulation of PTEN acetylation controls its localization and activity due to cellular damage and intracellular stress [26,33]. In our study, miR-138's effects on exosome-mediated wound healing were associated with inhibition of SIRT1 expression, and the downregulation expression of SIRT1 suppressed PTEN expression, but promotion phosphorylation of Akt and ERK1/2. The activation of $\mathrm{PI3K} / \mathrm{Akt}$ and ERK1/2 signaling reversed HG induced cell apoptosis (Figure. 70). Our study also found that miR-138 from ADSCs exosomes accelerates wound

Loading [MathJax]/jax/output/CommonHTML/fonts/TeX/fontdata.js 
healing in diabetic rats by promote angiogenesis. miR-183 overexpression activated the PI3K/Akt pathway which led to upregulation of downstream target genes including VEGF and CD34. Therefore, growth and angiogenesis of endothelial cells were promoted [43].

Exosomes are paracrine mediators that transfer proteins and genetic material to target cells. Exogenous exosomal molecules might regulate target protein or gene expression as well as recipient cell function [44,45]. For instance, colorectal cancer exosomes are used to transfer mRNAs that enrich endothelial cell proliferation and facilitate angiogenesis [46]. Exosomes derived from human umbilical cord MSCs have been demonstrated to transfer miR-181c and to attenuate burn-induced inflammation [47]. Human umbilical cord blood exosomes have been reported to enhance cutaneous wound healing via miR-21-3pmediated promotion regarding fibroblast function and angiogenesis [30].

\section{Conclusions}

In this study, miR-138 transferred by ADSC-exosomes mediated wound healing primarily through preventing cell death. The wound healing promoted by exosomes from ADSCs overexpressing miR-138 suggests that exosome transplantation may have potential clinical applications in diabetes-induced skin injury treatment.

\section{Abbreviations}

Exo, exosome, ADSCs, adipose-derived mesenchymal stem cells, HSFs, human scar fibroblasts, HMECs, human mammary epithelial cells, HG, high glucose, CCK-8, cell counting kit-8, 3'UTRs, 3' untranslated regions, SIRT1, sirtuin type 1, Sir2, silent information regulator 2, PBS, phosphate-buffered saline, DMEM, dulbecco's modified eagle's medium, FITC, fluorescein isothiocyanate, PE, phycoerythrin, von vWF, Willebrand factor, $\mathrm{PI}$, propidium iodide, TUNEL, terminal deoxynucleotidyl transferase dUTP nick end labeling.

\section{Declarations}

\section{Availability of data and materials}

Availability of data and materials can be assessed both in the "Methods" section, the "Results" section.

\section{Consent for publication}

Not applicable.

\section{Author Contributions}

$\mathrm{XL}, \mathrm{WZ}$ and $\mathrm{MZ}$ performed the experiments and analyzed the data, $\mathrm{JH}$ and $\mathrm{YS}$ conceived experiments and revised the draft, $\mathrm{HY}$ and $\mathrm{ML}$ conceived the studies and drafted the manuscript with feedback from all authors. All authors read and approved the final version.

\section{Competing interests}

There are no conflicts of interests.

\section{Acknowledgements}

None.

\section{Funding}

The study was aided by research grants from the China National Natural Science Foundation (No. 81671793) and Talents Training Program of Pudong Hospital affiliated to Fudan University (No. PX201705).

\section{Ethics approval and consent to participate}

The Animal Care and Utilization Committee of the Shanghai Tenth People's Hospital approved all animal procedures. We obtained male Sprague-Dawley female rats at eight weeks of age from the SLAC Laboratory Animal Co. Ltd, Shanghai, China, and individually housed them in independent ventilated cages under $24{ }^{\circ} \mathrm{C}$ to $26^{\circ} \mathrm{C}$ under constant humidity with a 12-hour light/dark cycle. All procedures were approved by the Ethics Committee of the the Shanghai Tenth People's Hospital, Shanghai, and were conducted by following the guidelines. We conducted surgical processes under anesthesia and made every effort to minimize suffering. We anesthetized rats by intraperitoneal injection of $30 \mathrm{mg} / \mathrm{kg}$ sodium pentobarbital before sacrifice and the rats were sacrifice by spinal dislocation.

\section{References}

1. Bustan RS, Wasim D, Yderstraede KB and Bygum A. Specific skin signs as a cutaneous marker of diabetes mellitus and the prediabetic state - a systematic review. Dan Med J. 2017, 64(1).

2. Chen Y, Ma Y, Li N, Wang H, Chen B, Liang Z, Ren R, Lu D, Boey J, Armstrong DG and Deng W. Efficacy and long-term longitudinal follow-up of bone marrow mesenchymal cell transplantation therapy in a diabetic patient with recurrent lower limb bullosis diabeticorum. Stem Cell Res Ther. $2018,9(1): 99$. 
3. Chang CJ, Yen ML, Chen YC, Chien CC, Huang HI, Bai CH and Yen BL. Placenta-derived multipotent cells exhibit immunosuppressive properties that are enhanced in the presence of interferon-gamma. Stem Cells. 2006, 24(11):2466-2477.

4. Bora P and Majumdar AS. Adipose tissue-derived stromal vascular fraction in regenerative medicine: a brief review on biology and translation. Stem Cell Res Ther. 2017, 8(1):145.

5. Kaisang L, Siyu W, Lijun F, Daoyan P, Xian CJ and Jie S. Adipose-derived stem cells seeded in Pluronic F-127 hydrogel promotes diabetic wound healing. J Surg Res. 2017, 217:63-74.

6. Seo E, Lim JS, Jun JB, Choi W, Hong IS and Jun HS. Exendin-4 in combination with adipose-derived stem cells promotes angiogenesis and improves diabetic wound healing. J Transl Med. 2017, 15(1):35.

7. Martin A, Komada MR and Sane DC. Abnormal angiogenesis in diabetes mellitus. Med Res Rev. 2003, 23(2):117-145.

8. Li X, Xie X, Lian W, Shi R, Han S, Zhang H, Lu L and Li M. Exosomes from adipose-derived stem cells overexpressing Nrf2 accelerate cutaneous wound healing by promoting vascularization in a diabetic foot ulcer rat model. Exp Mol Med. 2018, 50(4):29.

9. Robbins PD and Morelli AE. Regulation of immune responses by extracellular vesicles. Nat Rev Immunol. 2014, 14(3):195-208.

10. Thery C, Ostrowski M and Segura E. Membrane vesicles as conveyors of immune responses. Nat Rev Immunol. 2009, 9(8):581-593.

11. Bretz NP, Ridinger J, Rupp AK, Rimbach K, Keller S, Rupp C, Marme F, Umansky L, Umansky V, Eigenbrod T, Sammar M and Altevogt P. Body fluid exosomes promote secretion of inflammatory cytokines in monocytic cells via Toll-like receptor signaling. J Biol Chem. 2013, 288(51):36691-36702.

12. Kadiu I, Narayanasamy P, Dash PK, Zhang W and Gendelman HE. Biochemical and biologic characterization of exosomes and microvesicles as facilitators of HIV-1 infection in macrophages. J Immunol. 2012, 189(2):744-754.

13. Cabral J, Ryan AE, Griffin MD and Ritter T. Extracellular vesicles as modulators of wound healing. Adv Drug Deliv Rev. 2018, 129:394-406.

14. Kobayashi H, Ebisawa K, Kambe M, Kasai T, Suga H, Nakamura K, Narita Y, Ogata A and Kamei Y. Editors' Choice Effects of exosomes derived from the induced pluripotent stem cells on skin wound healing. Nagoya J Med Sci. 2018, 80(2):141-153.

15. Carrington JC and Ambros V. Role of microRNAs in plant and animal development. Science. 2003, 301(5631):336-338.

16. Engels BM and Hutvagner G. Principles and effects of microRNA-mediated post-transcriptional gene regulation. Oncogene. 2006, 25(46):6163-6169.

17. Mattes J, Collison A and Foster PS. Emerging role of microRNAs in disease pathogenesis and strategies for therapeutic modulation. Curr Opin Mol Ther. 2008, 10(2):150-157.

18. Taganov KD, Boldin MP and Baltimore D. MicroRNAs and immunity: tiny players in a big field. Immunity. 2007, 26(2):133-137.

19. Liu JJ, Zhang H, Xing F, Tang B, Wu SL, Xuan L, Kang PF, Xu Q, Wang HJ, Zhang NR and Wang XJ. MicroRNA138 promotes proliferation and suppresses mitochondrial depolarization in human pulmonary artery smooth muscle cells through targeting TASK1. Mol Med Rep. 2018, 17(2):3021-3027.

20. Zhang Y, Du X, Li W, Sang H, Qian A, Sun L, Li X and Li C. Resveratrol Improves Endothelial Progenitor Cell Function through miR-138 by Targeting Focal Adhesion Kinase (FAK) and Promotes Thrombus Resolution In Vivo. Med Sci Monit. 2018, 24:951-960.

21. Xiao YY, Fan PJ, Lei SR, Qi M and Yang XH. MiR-138/peroxisome proliferator-activated receptor beta signaling regulates human hypertrophic scar fibroblast proliferation and movement in vitro. J Dermatol. 2015, 42(5):485-495.

22. Tian F, Yuan C and Yue H. MiR-138/SIRT1 axis is implicated in impaired learning and memory abilities of cerebral ischemia/reperfusion injured rats. Exp Cell Res. 2018, 367(2):232-240.

23. Zhu J, Shi H, Liu H, Wang X and Li F. Long non-coding RNA TUG1 promotes cervical cancer progression by regulating the miR-138-5p-SIRT1 axis. Oncotarget. 2017, 8(39):65253-65264.

24. Wang $\mathrm{J}$ and Zhang $\mathrm{Y}$. Neuroprotective effect of berberine agonist against impairment of learning and memory skills in severe traumatic brain injury via Sirt1/p38 MAPK expression. Mol Med Rep. 2018, 17(5):6881-6886.

25. Wils J, Favre $\mathrm{J}$ and Bellien J. Modulating putative endothelial progenitor cells for the treatment of endothelial dysfunction and cardiovascular complications in diabetes. Pharmacol Ther. 2017, 170:98-115.

26. Chae HD and Broxmeyer HE. SIRT1 deficiency downregulates PTEN/JNK/FOXO1 pathway to block reactive oxygen species-induced apoptosis in mouse embryonic stem cells. Stem Cells Dev. 2011, 20(7):1277-1285.

27. Kim GD. Kaempferol Inhibits Angiogenesis by Suppressing HIF-1alpha and VEGFR2 Activation via ERK/p38 MAPK and PI3K/Akt/mTOR Signaling Pathways in Endothelial Cells. Prev Nutr Food Sci. 2017, 22(4):320-326.

28. Li M, Cheng W, Luo J, Hu X, Nie T, Lai H, Zheng X, Li F and Li H. Loss of selenocysteine insertion sequence binding protein 2 suppresses the proliferation, migration/invasion and hormone secretion of human trophoblast cells via the PI3K/Akt and ERK signaling pathway. Placenta. 2017, 55:81-89.

29. Zhang J, Chen C, Hu B, Niu X, Liu X, Zhang G, Zhang C, Li Q and Wang Y. Exosomes Derived from Human Endothelial Progenitor Cells Accelerate Cutaneous Wound Healing by Promoting Angiogenesis Through Erk1/2 Signaling. Int J Biol Sci. 2016, 12(12):1472-1487.

30. Hu Y, Rao SS, Wang ZX, Cao J, Tan YJ, Luo J, Li HM, Zhang WS, Chen CY and Xie H. Exosomes from human umbilical cord blood accelerate cutaneous wound healing through miR-21-3p-mediated promotion of angiogenesis and fibroblast function. Theranostics. 2018, 8(1):169-184.

31. Cui MD, Pan ZH and Pan LQ. Danggui Buxue Extract-Loaded Liposomes in Thermosensitive Gel Enhance In Vivo Dermal Wound Healing via Activation of the VEGF/PI3K/Akt and TGF-beta/Smads Signaling Pathway. Evid Based Complement Alternat Med. 2017, 2017:8407249.

32. Cui T, Jimenez JJ, Block NL, Badiavas EV, Rodriguez-Menocal L, Vila Granda A, Cai R, Sha W, Zarandi M, Perez R and Schally AV. Agonistic analogs of growth hormone releasing hormone $(\mathrm{GHRH})$ promote wound healing by stimulating the proliferation and survival of human dermal fibroblasts through ERK and AKT pathways. Oncotarget. 2016, 7(33):52661-52672.

Loading [MathJax]/jax/output/CommonHTML/fonts/TeX/fontdata.js 
33. Wang B, Wang D, Yan T and Yuan H. MiR-138-5p promotes TNF-alpha-induced apoptosis in human intervertebral disc degeneration by targeting SIRT1 through PTEN/PI3K/Akt signaling. Exp Cell Res. 2016, 345(2):199-205.

34. Wang Q, Tang H, Yin S and Dong C. Downregulation of microRNA-138 enhances the proliferation, migration and invasion of cholangiocarcinoma cells through the upregulation of RhoC/p-ERK/MMP-2/MMP-9. Oncol Rep. 2013, 29(5):2046-2052.

35. Xiong H, Luo T, He W, Xi Dan 1, Hao L, Li M, Liu J, Guo Z. Up-regulation of miR-138 inhibits hypoxia-induced cardiomyocyte apoptosis via down-regulating lipocalin-2 expression. Exp Biol Med (Maywood). 2016,241(1):25-30.

36. He S, Liu P, Jian Z, Li J, Zhu Y, Feng Z, Xiao Y. miR-138 protects cardiomyocytes from hypoxia-induced apoptosis via MLK3/JNK/c-jun pathway. Biochem Biophys Res Commun. 2013,441(4):763-9.

37. Zhang B, Wang M, Gong A, Zhang X, Wu X, Zhu Y, Shi H, Wu L, Zhu W, Qian H and Xu W. HucMSC-Exosome Mediated-Wnt4 Signaling Is Required for Cutaneous Wound Healing. Stem Cells. 2015, 33(7):2158-2168.

38. Chalhoub N and Baker SJ. PTEN and the PI3-kinase pathway in cancer. Annu Rev Pathol. 2009, 4:127-150.

39. Lee SB, Xuan Nguyen TL, Choi JW, Lee KH, Cho SW, Liu Z, Ye K, Bae SS and Ahn JY. Nuclear Akt interacts with B23/NPM and protects it from proteolytic cleavage, enhancing cell survival. Proc Natl Acad Sci U S A. 2008, 105(43):16584-16589.

40. Potocnjak I and Domitrovic R. Carvacrol attenuates acute kidney injury induced by cisplatin through suppression of ERK and PI3K/Akt activation. Food Chem Toxicol. 2016, 98(Pt B):251-261.

41. Tachibana N, Cantrup R, Dixit R, Touahri Y, Kaushik G, Zinyk D, Daftarian N, Biernaskie J, McFarlane S and Schuurmans C. Pten Regulates Retinal Amacrine Cell Number by Modulating Akt, Tgfbeta, and Erk Signaling. J Neurosci. 2016, 36(36):9454-9471.

42. Han Z, Chen Y, Zhang Y, Wei A, Zhou J, Li Q and Guo L. MiR-21/PTEN Axis Promotes Skin Wound Healing by Dendritic Cells Enhancement. J Cell Biochem. 2017, 118(10):3511-3519.

43. Zhang Z, Qin X, Zhang J. MicroRNA-183 inhibition exerts suppressive effects on diabetic retinopathy by inactivating BTG1-mediated PI3K/Akt/VEGF signaling pathway. Am J Physiol Endocrinol Metab. 2019, 316(6):E1050-E1060.

44. Park KC, Kim SM, Jeon JY, Kim BW, Kim HK, Chang HJ, Lee YS, Kim SY, Choi SH, Park CS and Chang HS. Synergistic Activity of N-hydroxy-7-(2naphthylthio) Heptanomide and Sorafenib Against Cancer Stem Cells, Anaplastic Thyroid Cancer. Neoplasia. 2017, 19(3):145-153.

45. Jiang N, Xiang L, He L, Yang G, Zheng J, Wang C, Zhang Y, Wang S, Zhou Y, Sheu TJ, Wu J, Chen K, Coelho PG, et al. Exosomes Mediate EpitheliumMesenchyme Crosstalk in Organ Development. ACS Nano. 2017, 11(8):7736-7746.

46. Hong BS, Cho JH, Kim H, Choi EJ, Rho S, Kim J, Kim JH, Choi DS, Kim YK, Hwang D and Gho YS. Colorectal cancer cell-derived microvesicles are enriched in cell cycle-related mRNAs that promote proliferation of endothelial cells. BMC Genomics. 2009, 10:556.

47. Li X, Liu L, Yang J, Yu Y, Chai J, Wang L, Ma L and Yin H. Exosome Derived From Human Umbilical Cord Mesenchymal Stem Cell Mediates MiR-181c Attenuating Burn-induced Excessive Inflammation. EBioMedicine. 2016, 8:72-82.

\section{Supplementary}

Supplementary Figure $\mathrm{S} 1$ is not available with this version

\section{Figures}


A

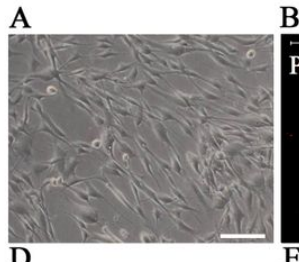

D

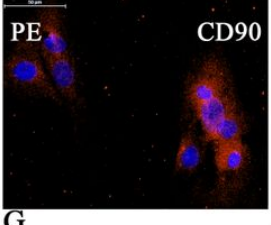

G
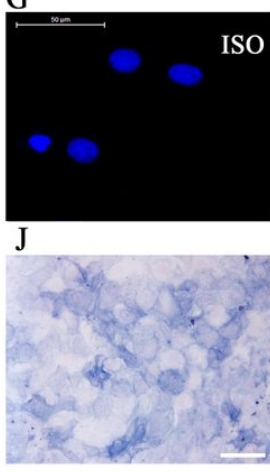

$\mathrm{L}$

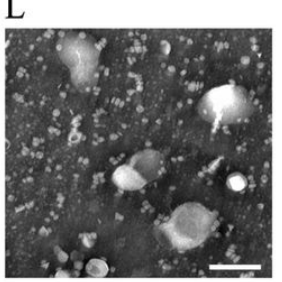

C
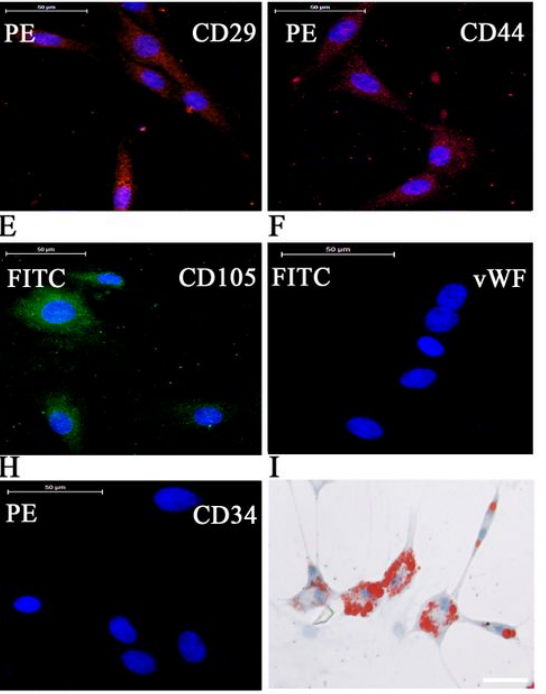

$\mathrm{K}$
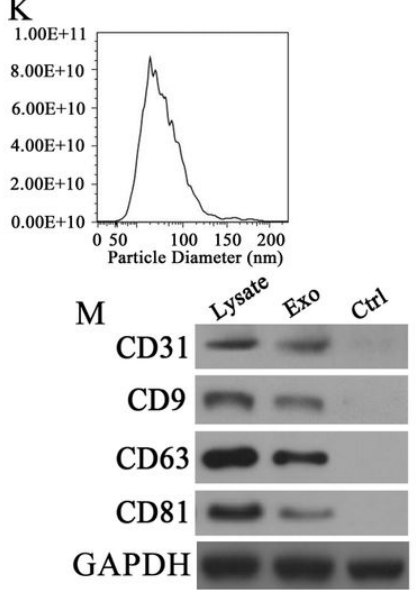

\section{Figure 1}

Characterization of exosomes released by adipose-derived mesenchymal stem cells. (A) ADSCs showed a typical cobblestone-like morphology. Scale bar: 50 $\mu \mathrm{m}$. (B-H) Immunofluorescence staining of cell surface markers. We labeled antibodies with either fluorescein isothiocyanate (FITC, green) or phycoerythrin (PE, red). Cell nucleus were staining with DAPI (blue). CD29, CD44, CD90, and CD105 are positive. CD34 and von Willebrand Factor (vWF) are negative. FITC and PE labeled mouse IgG isotype controls are shown ( $\times 200)$. Scale bar: $50 \mu \mathrm{m}$. (I and J) Differentiation potential of ADSCs assessed by oil red 0 (I) and alkaline phosphatase staining $(\mathrm{J})$. Scale bar: $30 \mu \mathrm{m}$. (K) Particle size distribution and concentration of ADSC-exosomes measured by tunable resistive pulse sensing. (L) Transmission electron micrographs showing ADSC-exosome morphology. Scale bar: $150 \mathrm{~nm}$. (M) Western blots of CD31, CD63, CD9, CD81 and GAPDH expression in exosomes from ADSCs. Ctrl, no exosome fraction, Exo, exosome fraction Lysate, cellular components. 
A

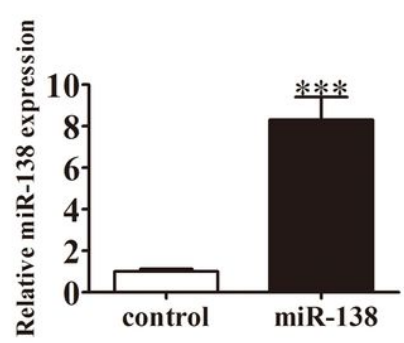

B

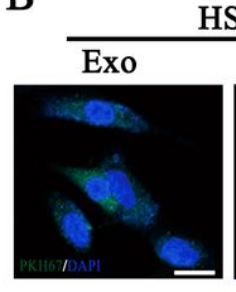

HSFs

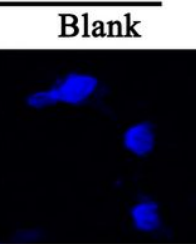

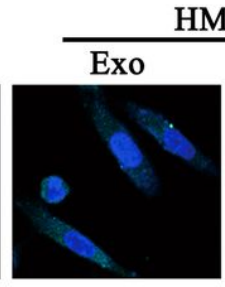

HMECs
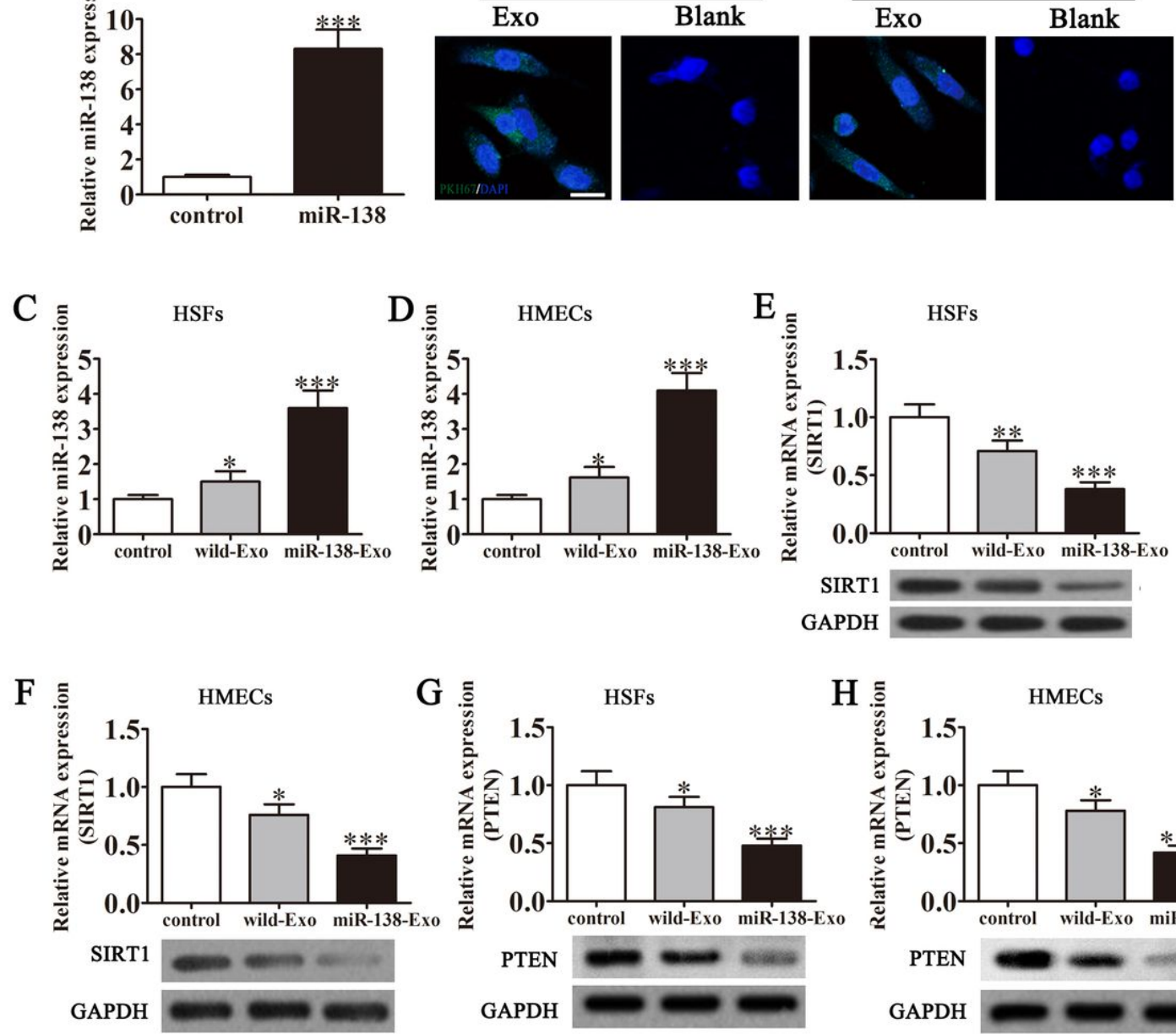

\section{Figure 2}

ADSC-exosomes deliver miR-138 into endothelial cells and fibroblasts. (A) qPCR assay of miR-138 expression in exosomes from ADSCs. ***p < 0.001 vs. control. (B) Fluorescence microscopy of PKH67-labeled ADSC-exosomes internalized by HSFs and HMECs. Green-labeled exosomes are visible in the perinuclear region of recipient cells. Scale bar: $20 \mu \mathrm{m}$. (C, D) After incubation with wild-type or miR-138 overexpressing ADSCs-exosomes (100 $\mu \mathrm{g} / \mathrm{ml})$ for $3 \mathrm{~h}$, miR-138 expression in HSFs (C) and HMECs (D) was increased. $n=3$. ${ }^{\star} p<0.05$, ${ }^{\star \star \star} p<0.001$ vs. controls. (E-H) SIRT1 and PTEN mRNA and protein expression in both HSFs and HMECs was decreased after incubation with wild-type or miR-138 overexpressing ADSC-exosomes $(100 \mu \mathrm{g} / \mathrm{ml})$ for $24 \mathrm{~h}$. $\mathrm{n}=3 .{ }^{*} \mathrm{p}$ $<0.05, * \star p<0.01, * \star \star p<0.001$ versus controls. 
A

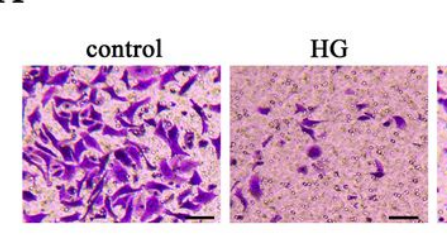

C
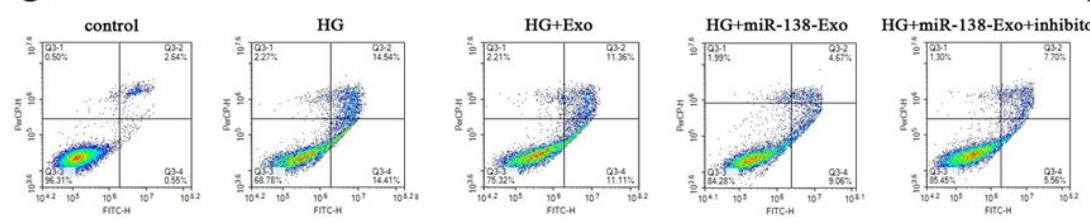

E
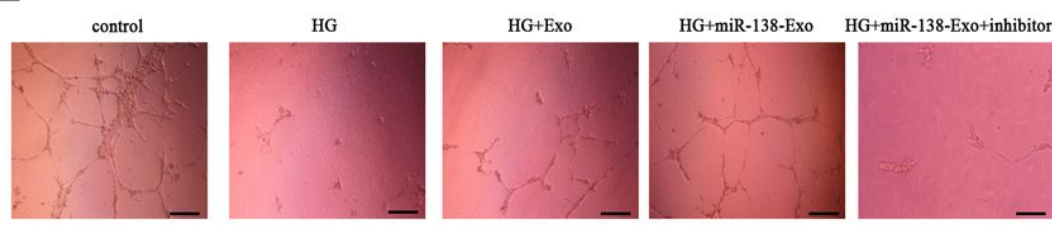

G

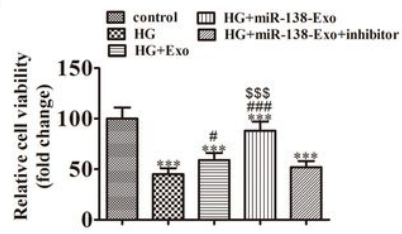

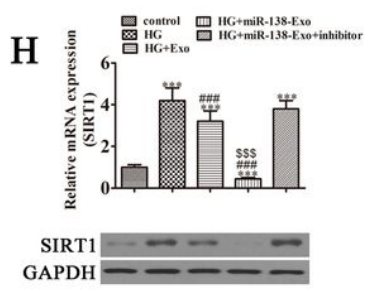

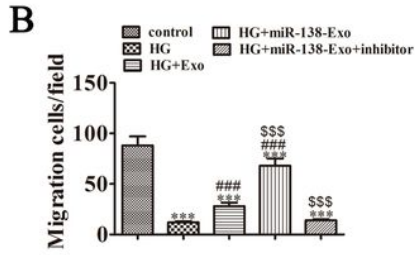

D

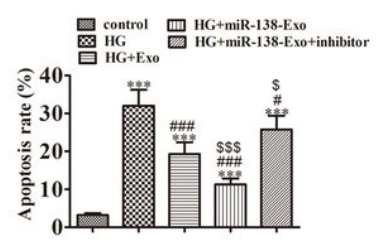

F
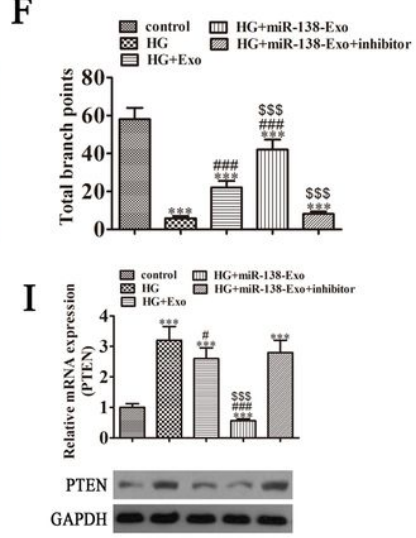

$\mathbf{J}$

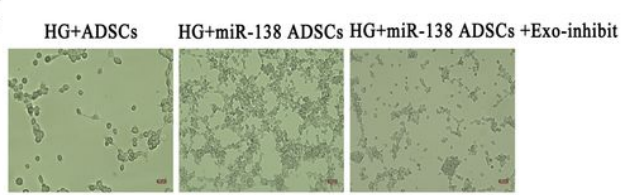

Figure 3

miR-138 was involved in ADSC-exosome-mediated angiogenesis, migration, and apoptosis inhibition of HMECs in cultures with 30 mM added glucose. (A) Representative photomicrographs of HMECs cells that migrated through the Transwell filter. Scale bar: $20 \mu \mathrm{m}$. (B) The cells in ten randomly selected fields were counted. $\mathrm{n}=3$. ${ }^{* \star *} \mathrm{p}<0.001 \mathrm{vs}$. control. \#\#\#p<0.001 vs. high glucose group.

$\$ \mathrm{p}<0.001$ vs. the high glucose plus exosome group. (C) We assayed HMEC apoptosis by flow cytometry after annexin V-FITC staining of cells cultured in $30 \mathrm{n}$

$\$ p<0.001$ vs. high glucose plus exosome group. (E, F) Representative photomicrographs of tube-like structures. Scale bar: $50 \mu \mathrm{m}$. Tube branch points were counted. $\mathrm{n}=10 .{ }^{* *} \mathrm{p}<0.001$ vs. control. \#\#\#p<0.001 vs. high glucose group.

$\$ p<0.001$ vs. high glucose plus exosome group. (G) The proliferation of HMECs with different treatments was determined by a CCK-8 assay. $\mathrm{n}=5 . * * * \mathrm{p}<0.0$

$\$ p<0.001$ vs. high glucose plus exosome group. $(\mathrm{H}, \mathrm{I})$ SIRT1 $(\mathrm{H})$ and PTEN $(\mathrm{I})$ mRNA and protein expression was decreased. $\mathrm{n}=3$. *** $<<0.001$ vs. control. \#p $<0.05$, \#\#\#p<0.001 vs. high glucose group. $\$ \$ \$ p<0.001 \mathrm{vs}$. high glucose plus exosome group. $(\mathrm{J})$ The tube formation capacity was measured co-culture assay between ADSCs and HMECs. 
A
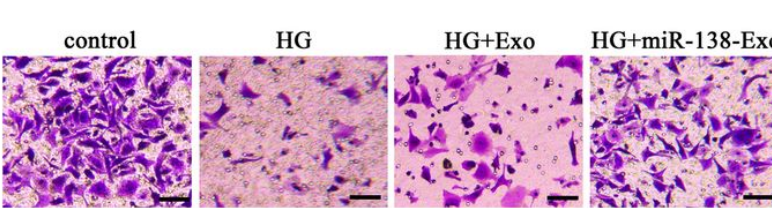

C

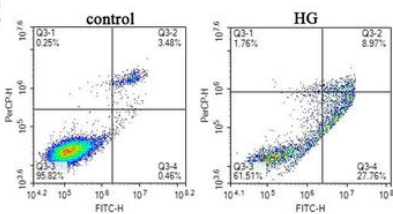

E

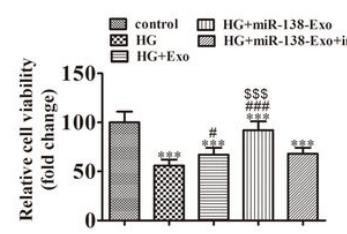

$\mathbf{H}$

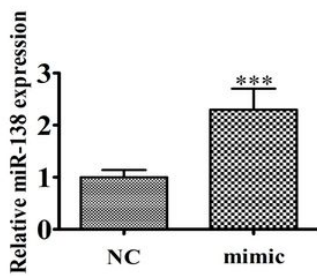

K

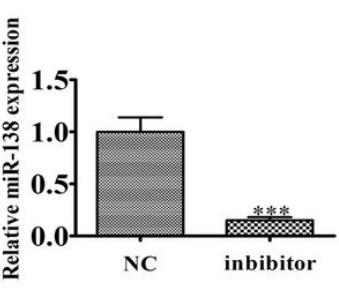

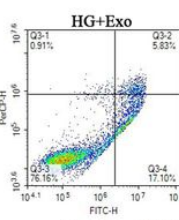

$\mathrm{F}$ 亭 冒

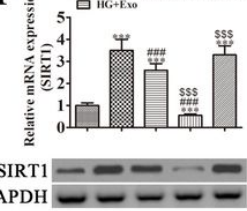

$\mathrm{HG}+\mathrm{miR}-138-\mathrm{Exo}$
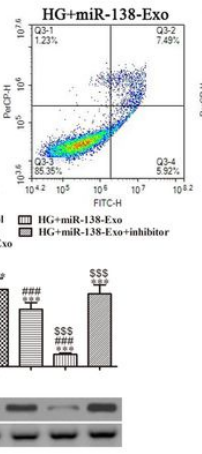

I.
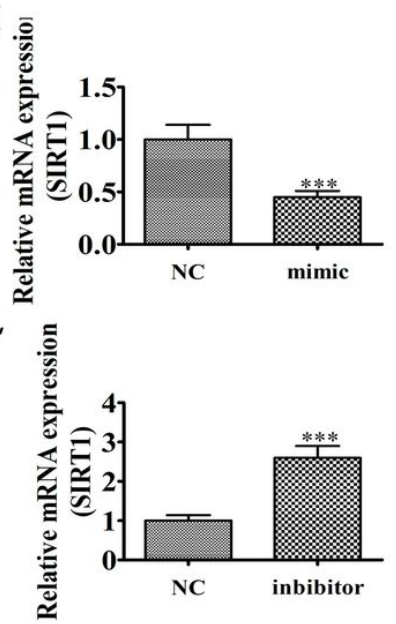

$\mathrm{HG}+\mathrm{miR}$-138-Exo +inhibitor

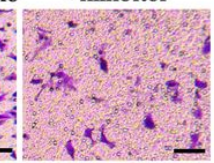

HG+miR-138-Exo
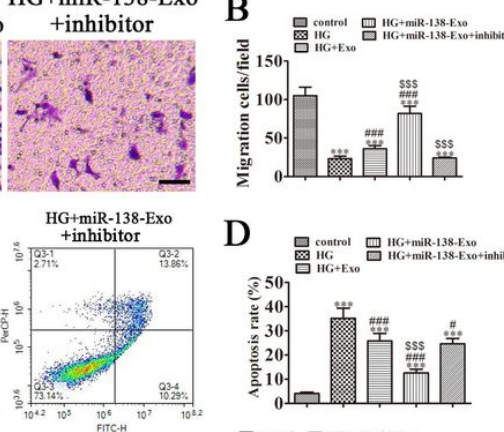

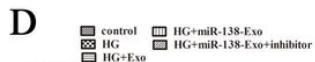

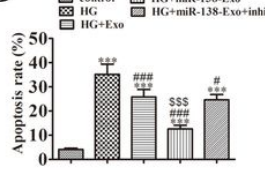

$\mathrm{G}$

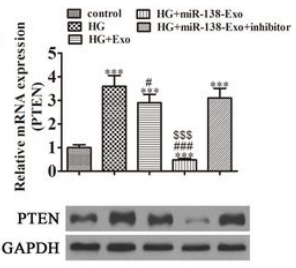

J

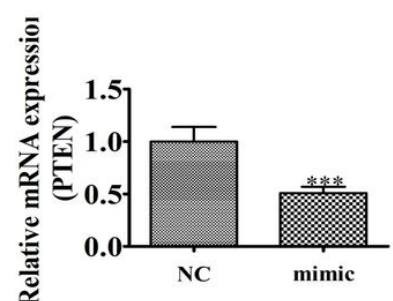

M

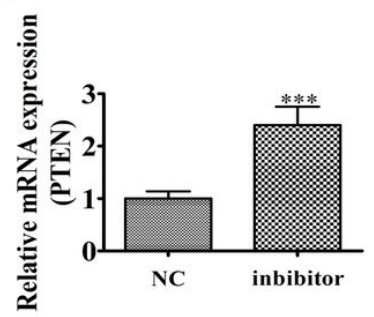

Figure 4

miR-138 was involved in ADSC-exosome-mediated activation and promotion of fibroblast migration. (A) Representative photomicrographs of HSFs that migrated through the Transwell filter. Scale bar: $20 \mu \mathrm{m}$. (B) The cells in ten randomly selected fields were counted. $n=3 . * \star \star p<0.001$ vs. control. \#\#\#p<0.001 vs. high glucose group.

$\$ \mathrm{p}<0.001$ vs. high glucose plus exosome group. (C) HSF apoptosis was assayed using flow cytometry after exposure to high glucose condition for $24 \mathrm{~h}$ and an $\$ p<0.001$ vs. the high glucose plus exosome group. (E) HSF cell proliferation following treatment was determined by a CCK-8 assay. $n=5 .{ }^{\star \star \star} \mathrm{p}<0.001$ vs. control. \#p $<0.05, \# \# \# p<0.001$ vs. the high glucose group.

$\$ \mathrm{p}<0.001$ vs. the high glucose plus exosome group. (F, G) SIRT1 (F) and PTEN (G) mRNA and protein expression were decreased. $\mathrm{n}=3 . * * * \mathrm{p}<0.001$ vs. con $\$ p<0.001$ vs. the high glucose plus exosome group. (H-M) RT-qPCR detection show the expression of miR-138, SIRT1, and PTEN in ADSCs after treansfected with miR-138 inhibitor or mimics. $n=5 .{ }^{\star \star *} p<0.001$ vs. NC. 
A

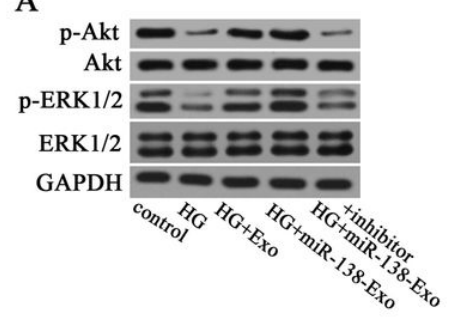

D

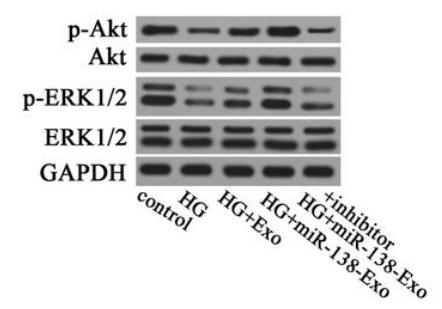

B

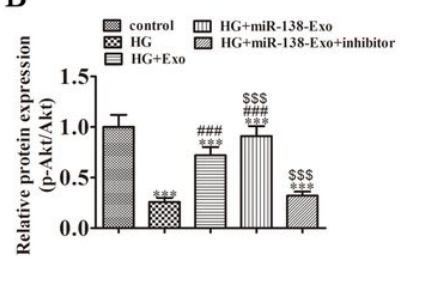

E

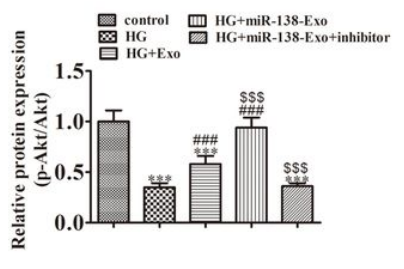

C

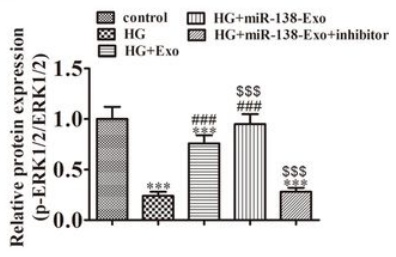

F

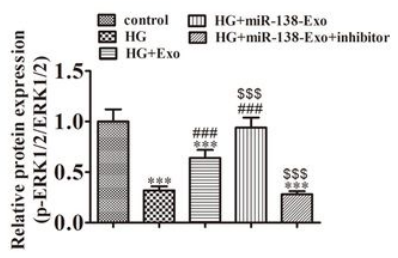

G

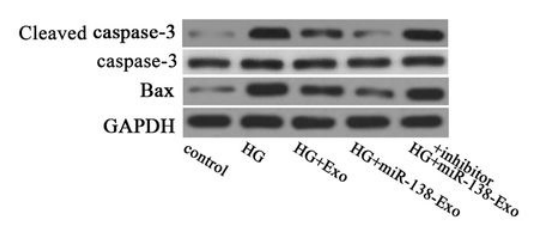

J

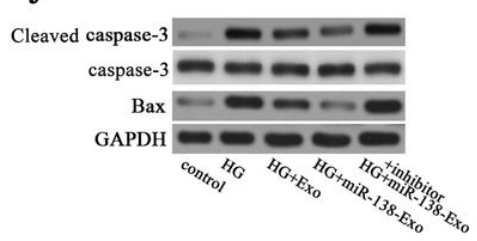

$\mathrm{H}$

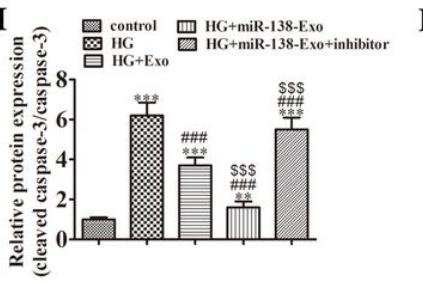

$\mathrm{K}$

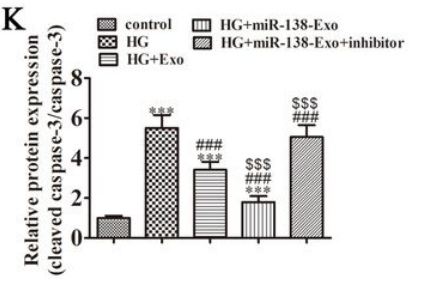

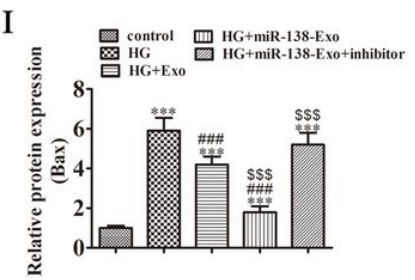

$\mathrm{L}$

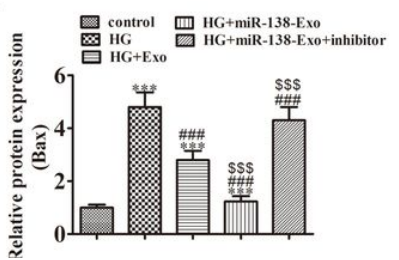

Figure 5

Akt and ERK1/2 phosphorylation was increased after intake of miR-138 from ADSC-exosomes. (A-C) Western blots of phosphorylated Akt and ERK1/2 expression in HMECs cells. $\mathrm{n}=3$. ${ }^{* *} \mathrm{p}<0.001$ vs. control. \#\#\#p 0.001 vs. high glucose group.

$\$ \mathrm{p}<0.001$ vs. high glucose plus exosome group. (D-F) Western blots of phosphorylated Akt and ERK1/2 expression in HSF cells. $\mathrm{n}=3$. $* *$ $\$ p<0.001$ vs. the high glucose plus exosome group. $(G-l)$ Western blots of caspase-3 and Bax apoptosis protein expression in HMEC cells. $n=3 . * \star * p<0.001$ vs. control. \#\#\# < 0.001 vs. the high glucose group.

$\$ \mathrm{p}<0.001$ vs. the high glucose plus exosome group. $(\mathrm{J}-\mathrm{L})$ Western blots of caspase-3 and Bax protein expression in HSF cells. $\mathrm{n}=3 . * * \mathrm{p}<$ $\$ p<0.001$ vs. the high glucose plus exosome group. 

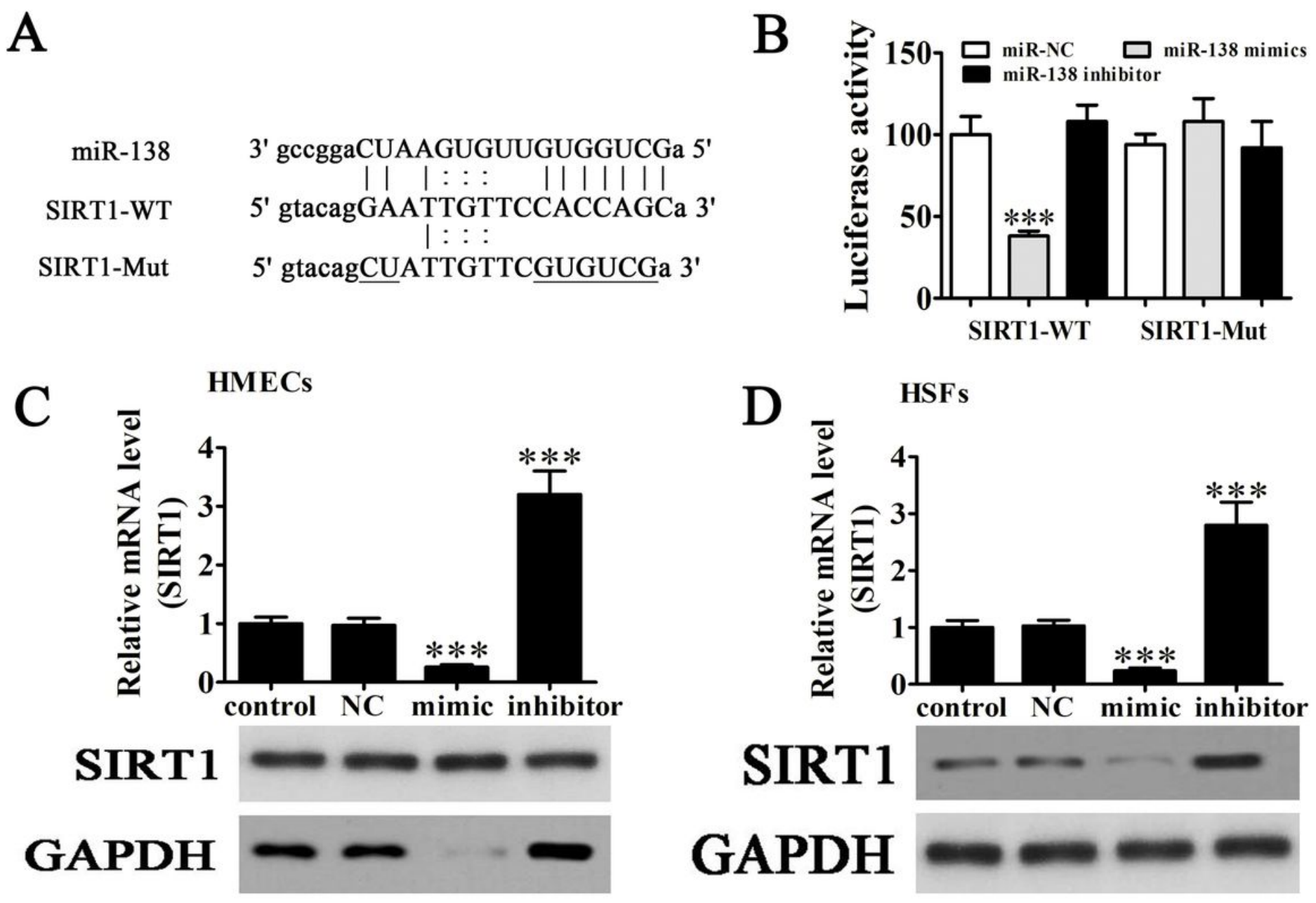

Figure 6

SIRT1 is a potential miR-138 target. (A) Complementary sequences in miR-138 and the SIRT1 mRNA 3'-UTR were obtained by publicly available algorithms. The mutated version of the SIRT1 3'-UTR is also given. (B) The SIRT1 3'-UTR was fused to the luciferase coding region (pMIR-SIRT1 3'-UTR) and cotransfected into 293T cells with miR-138 mimics to confirm that SIRT1 was the miR-138 target. The pMIR-SIRT1 3'-UTR and miR-138 mimic constructs were cotransfected

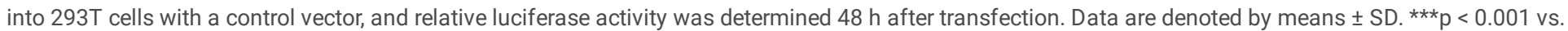
control. (C, D) Western blot and qPCR assays of SIRT1 expression in HMECs (C) and HSFs (D) after transfection with miR-138 mimics or inhibitor $(n=5)$. GAPDH expression the internal control. Data are means \pm SD. ${ }^{* *} p<0.001$ vs. the control. 
A

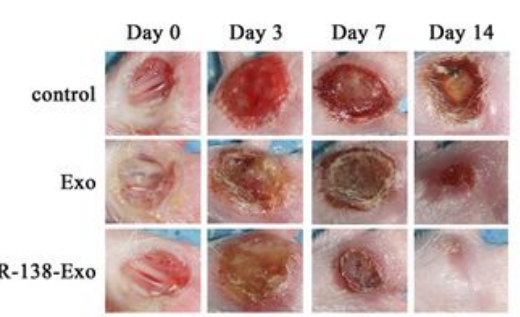

E

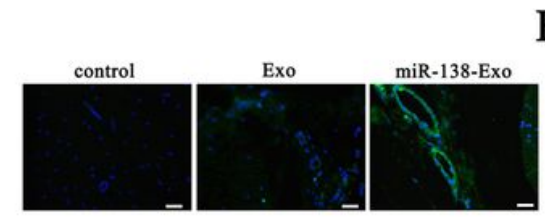

B

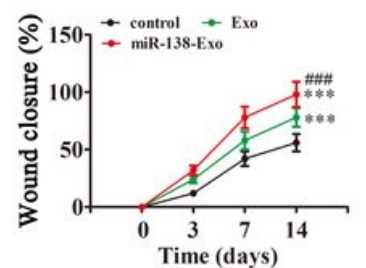

C

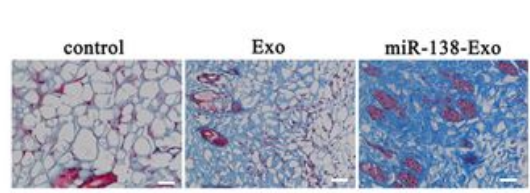

D

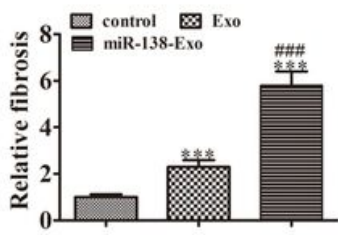

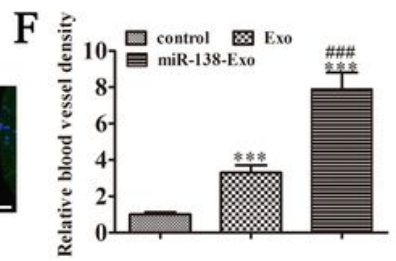

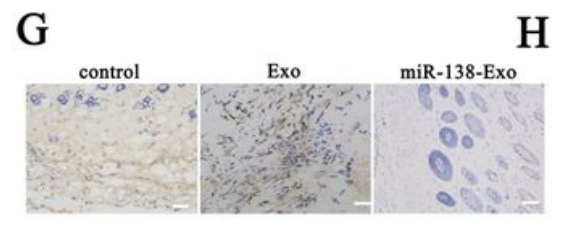

$\mathrm{H}$
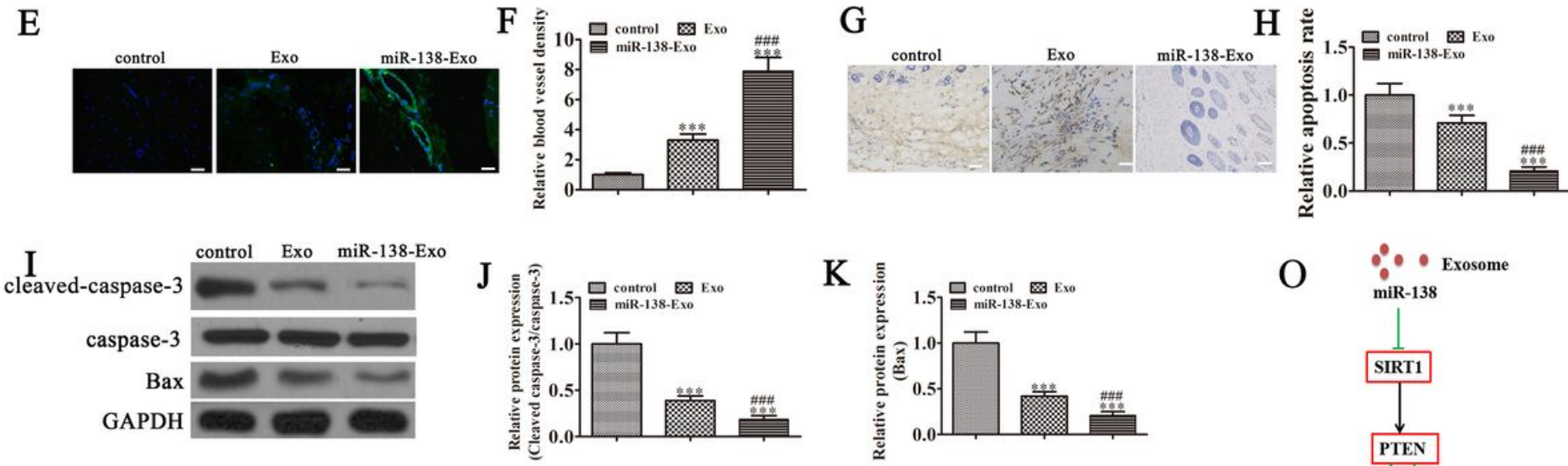

K

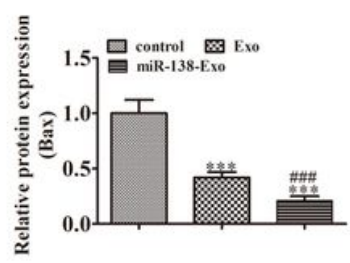

L

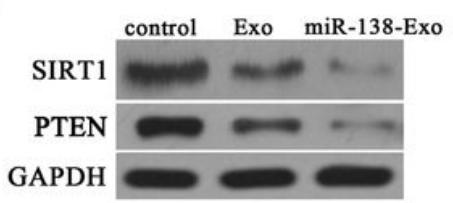

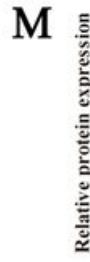

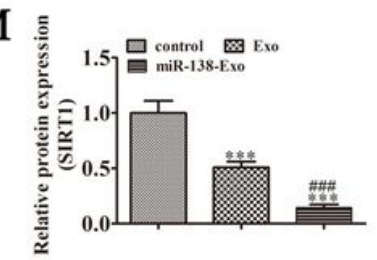

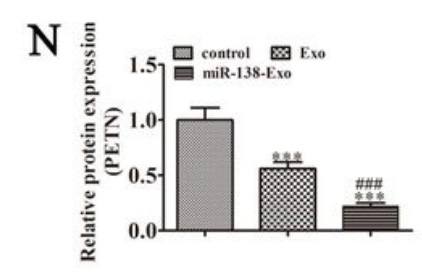

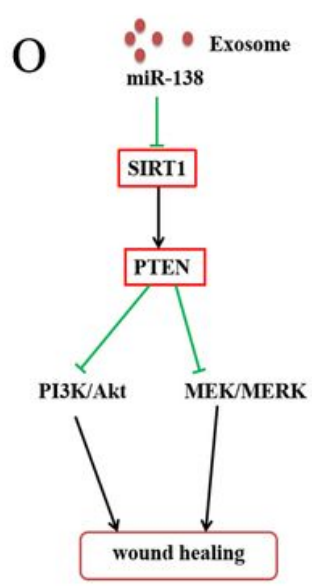

Figure 7

Exosomes from miR-138-modified ADSCs accelerated cutaneous wound healing in diabetic rats. (A, B) Representative images of full-thickness skin defects after treatment with ADSC-exosomes or ADSC-exosomes overexpressing miR-138 (miR-138-ADSC-exosomes) for 0, 3, 7, and 14 days after wounding . Percentage wound closure was calculated. $n=6$. ${ }^{* \star *} p<0.001$ vs. control. \#\#\#p $<0.001$ vs. exosomes. (C, D) Collagen deposition and tissue fibrosis were assessed by Masson trichrome staining after treatment with PBS or exosomes for 14 days. Scale bar: $30 \mu \mathrm{m}$. $n=6$. ${ }^{* * *} \mathrm{p}<0.001 \mathrm{vs}$. control. \#\#\#p $<0.001 \mathrm{vs}$. exosomes. (E, F) Immunofluorescence staining of microvascular formation with CD31 (FITC, green). Cell nucleus were staining with DAPI (blue). Scale bar: 30 $\mu \mathrm{m} . \mathrm{n}=6 .{ }^{\star \star \star} \mathrm{p}<0.001$ vs. control. \#\#\#p<0.001 vs. exosomes. $(\mathrm{G}, \mathrm{H})$ Apoptosis level was assayed by TUNEL staining. Scale bar: $30 \mu \mathrm{m}$. $n=6$. ${ }^{\star \star *} \mathrm{p}<0.001 \mathrm{vs.}$ control. \#\#\#p $<0.001$ vs. the exosomes. (I-K) Western blots cleaved caspase-3 and Bax protein expression. $n=6$. ${ }^{\star \star \star} p<0.001 \mathrm{vs}$. control. \#\#\#p $<0.001$ vs. exosomes. $(\mathrm{L}-\mathrm{N})$ Western blots of SIRT1 and PTEN protein expression. $\mathrm{n}=6$. ${ }^{\star \star \star} \mathrm{p}<0.001 \mathrm{vs}$. control. \#\#\#p<0.001 vs. exosomes. (0) a brief graphical representation. 